\title{
Surgical debulking, lymphatico venous anastomosis, vascularised lymph node transfer in lower limb lymphoedema
}

\author{
Hari Venkatramani, Rajasabapathy Raja Shanmugakrishnan, Murugesan Senthil Kumaran, \\ Shanmuganathan Raja Sabapathy
}

Plastic, Hand \& Reconstructive Microsurgery \& Burns, Ganga Hospital, Coimbatore 641043, India.

Correspondence to: Hari Venkatramani, Plastic, Hand \& Reconstructive Microsurgery \& Burns, Ganga Hospital, Coimbatore 641012, India. E-mail: drhariv@gmail.com

How to cite this article: Venkatramani H, Shanmugakrishnan RR, Kumaran MS, Sabapathy SR. Surgical debulking, lymphatico venous anastomosis, vascularised lymph node transfer in lower limb lymphoedema. Plast Aesthet Res 2020;7:19. http://dx.doi.org/10.20517/2347-9264.2019.70

Received: 7 Dec 2019 First Decision: 4 Mar 2020 Revised: 30 Mar 2020 Accepted: 7 Apr 2020 Published: 17 Apr 2020

Science Editor: Xiao Long Copy Editor: Jing-Wen Zhang Production Editor: Tian Zhang

\begin{abstract}
Lymphoedema is a chronic debilitating disease of the lymphatic system that occurs due to either abnormal development or damage of the lymphatics resulting from cancer or infection. The optimal treatment of lymphoedema is still elusive. Management is tailored according to clinical features, investigations and expectations of each patient. Lymphoedema patients should undergo a trial of conservative management with compression therapy, manual lymphatic drainage and external sequential compression devices. Early lymphoedema is treated by lymphovascular anastomosis, where the lymph vessels are connected to the subdermal veins by supermicrosurgery. In late cases when the limb is fibrotic, vascularised lymph node transfers are done, where lymph nodes are transferred from a healthy area to the affected area. In advanced cases, when the limb is fibrotic with cutaneous folds and skin changes, surgical debulking is done. In lymphoedema, along with accumulation of lymphatic tissue, there is also fat deposition, which can be removed by liposuction. One should be conversant with all treatment modalities to provide the lymphoedema patient with optimal care.
\end{abstract}

Keywords: Lymphoedema, lymphovascular anastomosis, vascularised lymph node transfers, liposuction, surgical debulking

\section{INTRODUCTION}

Lymphoedema is a chronic debilitating disease of the lymphatic system that affects more than 250 million people worldwide ${ }^{[1]}$. Lymphoedema results from abnormal development or damage of the lymphatics due 
to various causes such as filariasis or more commonly due to the various cancer treatment modalities. The resultant accumulation of lymphatic fluid causes persistent swelling of the limb, fat hypertrophy, tissue fibrosis, ulceration, infection and sepsis. Lymphoedema progresses through four stages ${ }^{[2]}$. Stage 0 indicates a clinically normal limb, but with abnormal lymph transport. Stage 1 shows early pitting oedema which improves with limb elevation. In stage 2, there is limb oedema that does not improve with limb elevation. Stage 3 represents pitting limb oedema with skin changes such as acanthosis, further deposition of fat and fibrosis and warty overgrowths. Currently, there is no cure for lymphoedema, and the optimal treatment is yet to be determined. The treatment for lymphoedema starts with conservative management followed by surgery if necessary. The various surgical modalities for lymphoedema are detailed in this review.

Lymphoedema management should ideally start with non-surgical modalities such as compression therapy, lymphoedema-specific manual lymphatic drainage and use of external sequential pneumatic compression devices ${ }^{[3]}$. This would not only reduce the amount of lymphoedema but would also facilitate surgical procedures by making the skin more pliable and soft. Lee recommends using non-surgical modalities for 2 years before attempting any surgical procedure ${ }^{[4]}$. The patient should be encouraged and counselled regarding the importance of conservative measures which can be difficult to achieve. Compliance is a problem as it can be costly, time-consuming and uncomfortable ${ }^{[5]}$.

Patients with early-stage lymphoedema benefit from physiological procedures such as lymphovenous anastomosis (LVA), where the lymphatics are connected to the veins or by vascularised lymph node transfers (VLNTs), in which lymph nodes from one part of the body are transferred to the affected area to drain excess lymphatic fluid. Liposuction is done for those patients whose swelling is mainly due to excess fat. For patients with end-stage disease and large fibrotic limbs, conservative therapy can be ineffective, and excisional surgery alone or in combination with other physiological procedures such as VLNT and LVA, is performed.

\section{LVA}

LVA is a microsurgical approach used to augment the return of lymph to the blood circulation in which lymphatic vessels smaller than $0.8 \mathrm{~mm}$ are connected to the subdermal venules using fine microsurgical sutures, instruments and high resolution magnification microscopes ${ }^{[6]}$. LVA is used to treat early stage lymphoedema. Chang et al. ${ }^{[7]}$ found that LVA was more effective when used for early stage lymphoedema (Stages $1 \& 2$ ) when compared to late stage lymphoedema (Stages $3 \& 4$ ) according to the M.D. Anderson Classification based on indocyanine green (ICG) lymphangiography. They postulated that this observation was because LVA needs intact functional lymphatic channels and minimal irreversible tissue fibrosis for it to be effective. In later stages, LVA could be combined with VLNT.

The patient is advised to wear compression stockings without exception during the day for at least 6 months to reduce oedema before LVA is attempted. The stockings are to be replaced with a new pair if they get loose. The patients are also told that they would have to continue wearing the compression stockings even after surgery to get the best results. Although LVA can be done without ICG lymphangiography, this imaging technique helps to assess the severity of lymphoedema and determine whether LVA would be effective, and it also aids in localising the position of the lymphatic channels in the limb ${ }^{[8]}$. The position of the lymphatic channels is marked using a pen on the skin. ICG lymphography can detect the position of the lymphatic channels only up to a depth of $10 \mathrm{~mm}$ from the skin surface. LVA can be done under regional or general anaesthesia under tourniquet control, or it can be done with a local anaesthetic containing adrenaline to limit bleeding from the dermal edges. A $3-\mathrm{cm}$ incision is made where the lymphatic ducts are located by ICG lymphography. Although there is no consensus on the number of anastomoses needed to obtain a significant reduction in lymphoedema, it is believed that increasing the number of LVA can improve lymphoedema better ${ }^{[9]}$. If ICG lymphography is not available, 5 to 10 incisions can be made 
on the medial and lateral side of the affected extremities as the lymphatics are more common in these areas. Isosulfan blue (Lymphazurin; Covidien) is injected just distal to the incision site. The dye easily gets absorbed into the lymphatic channels and facilitates easy visualisation of the lymphatic channels. In advanced lymphoedema, the visualisation of the dye can be less due to weaker staining because of reduced transport of the dye ${ }^{[10]}$. Small subdermal venules less than $0.5 \mathrm{~mm}$ with no backflow are preferably chosen for LVA since they have low intravascular pressure. Larger veins with backflow are associated with higher intravascular pressure and obstruction of the anastomotic site.

Recently, magnetic resonance lymphangiography (MRL) has been added to the armamentarium to help localise the lymphatic channels and venules for $\mathrm{LVA}^{[11]}$. A mixture of paramagnetic contrast medium containing gadobenate dimeglumine and lignocaine is injected subcutaneously/intradermally in the webspaces of both feet and MRI is performed. MRL diagnoses lymphoedema, grades the lymphoedema on the basis of the lymphatic drainage pattern and the delay of drainage, provides the number, diameter, course, and depth from the skin of both affected lymphatic vessels and the nearest veins, the distance between the lymphatics and the venules, and the location of the lymph nodes. Although the spatial orientation of the lymphatics that MRL provides along with no radiation is a highlight, it has some disadvantages in that it is costly, not freely available and time-consuming, and has occasional difficulty in distinguishing the affected lymphatic vessel from the adjacent vein when there is dye contamination in the venous system.

Once the appropriate lymphatic vessels and veins are identified, anastomosis is done between the lymphatic channels and the veins. This can be a technical challenge as the veins and the lymphatics are very small. Special instruments, sutures finer than 10-0 and high resolution microscopes with magnification of $20 \times$ to $30 \times$ are needed to perform this procedure. The anastomosis between lymphatics and veins are most commonly done in an end-to-end manner. The lymphatic vessel is smaller and thinner and often collapses, making it difficult to place the needle in the vessel. To increase the size of the lymphatic vessel, Yamamoto et al. ${ }^{[12]}$ proposed clamping the proximal lymphatics and massaging distally. Narushima et al. ${ }^{[13]}$ proposed using a 6-0 prolene suture as a stent within the lymphatic vessel to prevent suturing the backwall during anastomosis. When the vein is much smaller than the lymphatic vessel, the end of the vein can be sutured to the side of the lymphatic vessel in a side-to-end manner ${ }^{[14]}$. There is also a concern that the higher pressure in the vein can lead to obstruction of the anastomosis. Some proponents believe that the most optimal orientation would be to anastomose the end of the vein to the side of the lymphatic vessel, thus allowing the bidirectional flow of lymphatics to the veins ${ }^{[15]}$. This could also be achieved by anastomosing both the proximal and the distal cut ends of the lymphatics to veins ${ }^{[16]}$. Chen et al ${ }^{[17]}$ have proposed the "octopus lymphovascular anastomosis technique", where multiple small lymphatic channels are anastomosed to a single vein. A 12-0 suture is placed transluminally on the vein and then through the adventitia only on the lymphatic vessels which will cause intussusception of the lymphatic vessels into the vein. After anastomosis, the patency and the flow direction can be assessed by using patent blue dye or ICG.

Postoperatively, Koshima advocates using an infusion of a vasodilator drug (prostaglandin E1) for 5 days and then using an oral form for several weeks ${ }^{[10]}$. As the incisions are only skin deep, the patient usually has very little pain and can go home the same day. Bandages are applied until the wounds heal. Compression stockings are used for 3 weeks after the operation and continued for at least 6 months after the procedure.

Scaglioni et al ${ }^{[18]}$ reviewed 18 studies of LVA involving 939 patients and found that all studies showed objective reduction in the circumference measurement. Subjective symptom relief was found in $50 \%$ to $100 \%$ of patients as well as reduction in the cellulitis episodes in all cases. Several studies also show a striking reduction in the episodes of cellulitis post-surgery ${ }^{[19-21]}$. Results of LVA were noted by Chang et al. ${ }^{[7]}$ to be better in the upper limb (96\% symptomatic improvement) compared to the lower limb (57\% 

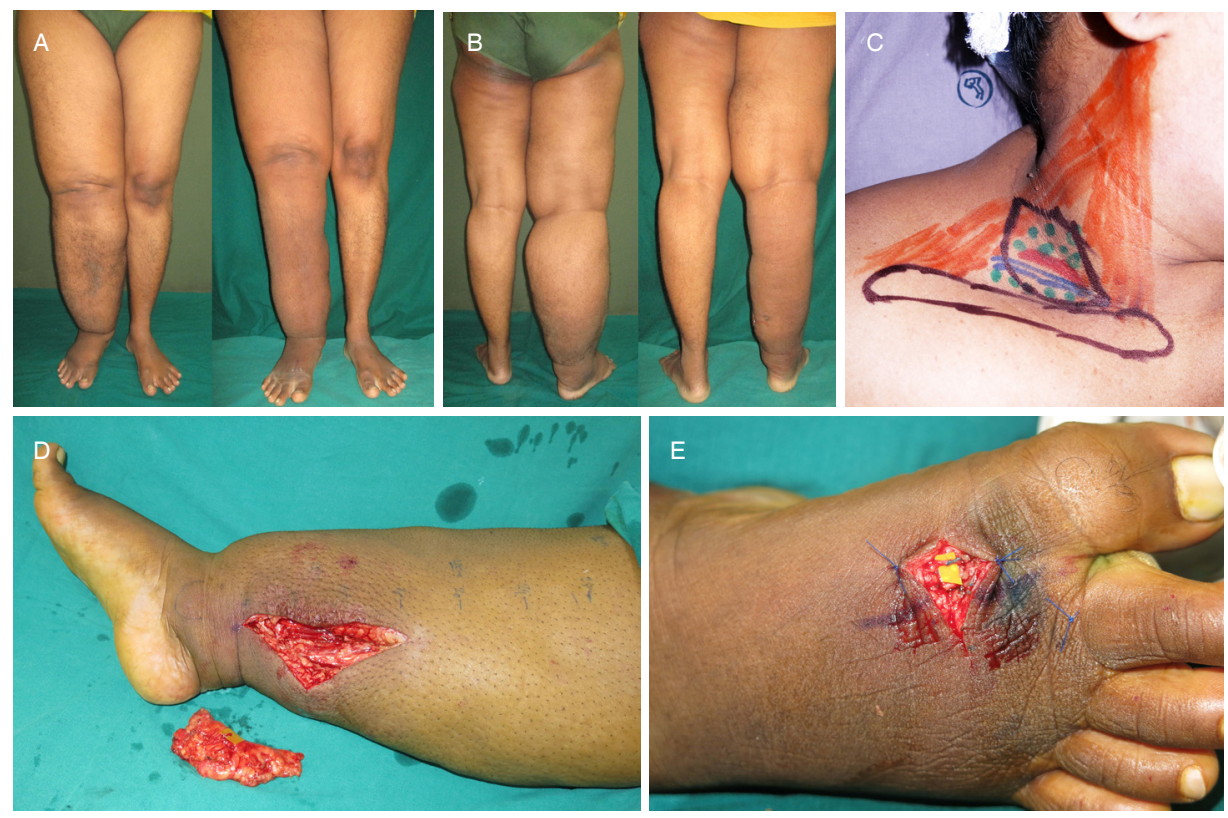

Figure 1. A, B: pre-op and Post-op photo of a patient with lymphoedema of the right leg; C, D: supraclavicular flap harvested and anastomosed to the posterior tibial artery; E: lymphovascular anastomosis done in the right foot

symptomatic improvement), since the lower limb is dependent and associated with higher venous pressures. The efficacy of LVA treatment is dependent more on the severity of the lymphoedema than to the duration of the lymphedema, with later stage lymphedema being less responsive.

\section{VLNT}

VLNT is a means to treat lymphoedema by replenishing the missing lymph nodes of the affected extremity by harvesting healthy vascularised lymph nodes from one area of the body and transplanting them to the affected extremity with the help of microsurgery ${ }^{[22]}$.

In early cases of lymphoedema, LVA is possible. However, in late cases, the limb is fibrotic and sclerosed, and VLNT is a physiological means to improve lymphoedema. The presence of significant backflow with few or no functioning lymphatic vessels on imaging using ICG, lymphography, lymphoscintigraphy or MRL suggests that VLNT may be indicated ${ }^{[23]}$. VLNT can be combined with LVA in certain cases if some good lymphatic channels are available, to give a better outcome [Figure $1 \mathrm{~A}-\mathrm{E}]$.

Several theories regarding how VLNT works have been postulated, since the precise mechanism of action of VLNT is incompletely understood. Honkonen et al. ${ }^{[24]}$ used a swine model to propose that VLNT acts like a "lymphatic wick" between the proximal and distal lymphatic vessels at the recipient site. This theory seems to be attractive, especially in the early stages when functional lymphatic channels are retained in both the proximal and distal segment. It is also believed that lymphatic connections are established between the vascularised lymph node flaps and the surrounding tissues. Saaristo proposed that high levels of vascular endothelial growth factor $\mathrm{C}$ is produced by the transferred lymph nodes which induces lymphangiogenesis and facilitates recanalisation of the lymphatic vessels between the recipient and transferred lymph nodes ${ }^{[25]}$. These theories probably explain why VLNTs are transferred to proximal levels in the limbs. This is best illustrated by the case of using the deep inferior epigastric perforator (DIEP) flap along with the lymph nodes in the groin to treat post-mastectomy lymphoedema. Besides, the release of scar tissue in the axilla can result in early improvement by releasing the pressure on the axillary vein which will reduce capillary filtration. There is also abundant soft tissue to allow easy closure at the donor site, and the scar is well 
Table 1. Advantages and disadvantages of various types of vascularised lymph node transfers

\begin{tabular}{|c|c|c|}
\hline $\begin{array}{l}\text { Vascularised } \\
\text { lymph node flaps }\end{array}$ & Advantages & Disadvantages \\
\hline Groin & $\begin{array}{l}\text { Can be taken with DIEP Flap during breast } \\
\text { reconstruction; well concealed scar; good cosmesis; } \\
\text { commonly used for upper limb lymphoedema }\end{array}$ & latrogenic lower limb lymphoedema \\
\hline Omentum & $\begin{array}{l}\text { No iatrogenic lymphoedema; rich source of } \\
\text { lymphatic tissue; }\end{array}$ & $\begin{array}{l}\text { Laparoscopy/laparotomy needed; poor cosmesis; complications due to } \\
\text { laparotomy; adhesions; hernias; DVT }\end{array}$ \\
\hline Submental & Less iatrogenic lymphoedema & $\begin{array}{l}\text { Injury to marginal mandibular branch of facial nerve; vessel is small; few } \\
\text { nodes in the flap }\end{array}$ \\
\hline Supraclavicular & Less iatrogenic lymphoedema & Vessel is small; few nodes; damage to brachial plexus and lymphatic duct \\
\hline Lateral thoracic & Commonly used for lower limb lymphoedema & latrogenic upper limb lymphoedema; damage to the thoracodorsal nerve \\
\hline Jejunum & No iatrogenic lymphoedema & Injury to the viscera, bowel adhesions, internal hernia; bowel ischaemia \\
\hline
\end{tabular}

DIEP: deep inferior epigastric perforator; DVT: deep vein thrombosis

hidden. If it is difficult to access the scar in the anatomical position, then the VLNTs can be placed just distal to the point of obstruction. For example, in patients who have had pelvic lymph nodes removed after a laparotomy, the vascularised lymph nodes would then be placed in the upper thigh medial to the femoral artery and the saphenous vein ${ }^{[1]}$.

Vascularised lymph nodes can also be transplanted distally in the limb. The theory behind placing the VLNT at non-anatomical sites is that they work like a "lymphatic pump". The strong arterial pulsations in the flap provide a strong hydrostatic force in the flap. The flap veins, which have low pressure, act like a suction drawing the lymphatic fluid into the capillaries ${ }^{[26]}$. Due to gravity, the lymphatic collection is predominantly distal, and placing the VLNTs distally seems to have a "catchment effect", thereby improving lymphatic drainage. However, distal placement of the flap can make the flap look bulky and non-aesthetic ${ }^{[27]}$. There are many potential donor lymph nodes for VLNTs, namely the groin, thoracic, submental, supraclavicular, omental and mesenteric lymph nodes. The advantages and disadvantages of the different donor options are listed in Table 1.

Breast cancer is the most common cancer among women and post-mastectomy lymphoedema occurs in $9 \%-41 \%$ of women who undergo axillary dissection and $4 \%-10 \%$ of women who undergo sentinel lymph node biopsy ${ }^{[28-30]}$. In such cases, the DIEP flap is the most commonly used. This flap not only helps in lymphoedema, but also helps to reconstruct the missing breasts at the same time. The superficial lymph node basin of the groin drains the lower abdomen and is the target for lymph node harvest, whereas deeper lymph nodes close to the femoral vessels drain the thigh and lower extremity. While harvesting the lymph nodes, it is preferable to harvest the lymph nodes lateral to the femoral vessels, since lymphatic drainage of the lower limb is predominantly medial to the femoral vessels and below the inguinal ligament ${ }^{[31]}$. The DIEP flap is raised as caudally as possible. The groin lymph nodes lateral to the femoral vessels are preferably harvested along with the superficial circumflex iliac vessels and are placed in the axilla, and the superficial circumflex iliac vein is anastomosed to either the thoracodorsal vein, the lateral thoracic vein or the serratus branch. The pedicle of the DIEP flap is attached either to the thoracodorsal vessels or the internal mammary vessels. The major concern about this flap is about donor site lymphoedema and can be prevented by reverse lymphatic mapping using preoperative lymphoscintigraphy, ICG and methylene blue dye ${ }^{[32]}$.

The supraclavicular flap based on the transverse cervical vessels can be used as a VLNT. Donor site lymphoedema is not common in this flap, as evidenced by the fact that the lymph nodes in this area are commonly removed during elective neck dissections after cancer ${ }^{[33]}$. The other benefit of the flap is that it generally heals well with a good scar. The flap dimensions and size are generally small compared to other donor sites, and this flap is generally placed distally in the limbs to treat lymphoedema, since the soft tissue cover around the wrist and ankles is much less. Safe harvesting of the supraclavicular flap would need 
thorough knowledge of the local anatomy and the consequent complications that can arise. The transverse cervical vessels can have a variable course. It can take its origin from either the thyrocervical trunk (80\%) or directly from the subclavian artery $(20 \%)$ or rarely from the internal mammary artery ${ }^{[34]}$. Careful surgical technique is necessary to avoid damage to the carotid artery, internal jugular vein, phrenic nerve and the thoracic duct ${ }^{[35]}$. The other concern about this flap is that it is thought to have fewer lymph nodes that can be transferred ${ }^{[36]}$.

The submental flap based on the submental artery is a commonly used flap for head and neck reconstruction ${ }^{[37]}$. This flap can be raised as a free flap containing the submental lymph nodes and used to treat upper and lower limb lymphoedema. Similar to the supraclavicular flap, removal of the lymph nodes in the neck is inconsequential regarding donor site lymphoedema as evidenced by their routine harvest during oncological lymph node dissections. A small elliptical skin paddle can also be included. The upper border of the incision is along the lower border of the mandible and extends from the angle to the symphysis. Dissection is performed deep to the platysma. The anterior belly of the digastric muscle can be included to avoid damage to the perforators supplying the flap. Soft tissue around the junction of the submental and facial vessels is included to provide more lymph nodes in the neck. The main concern regarding the submental flap is the possible injury to the marginal mandibular nerve that one should be wary of while raising the flap ${ }^{[38]}$. Furthermore, the pedicle can be very short, which may need including the facial vessels to make it longer ${ }^{[39]}$.

The lateral thoracic flap involves the transfer of lymphatics between the anterior and posterior axillary folds lateral to the pectoralis minor ${ }^{[40]}$. The dominant vascular supply to these nodes is from the lateral thoracic vessels. The artery can be absent in $12.5 \%$ of cases, in which case the thoracodorsal vessels provide the vascular supply to these nodes ${ }^{[40]}$. The main advantage of this flap is the inconspicuous scar which is well hidden in the axillary fold and the longer pedicle length when compared to the other peripheral lymph node flaps. The main disadvantage of the flap is that lymphoedema can occur in the potential donor site in the upper limb.

The greater omentum can be harvested as a free flap based on the gastroepiploic vessels to treat lymphoedema. The omentum has abundant lymph nodes and helps to initiate absorption from the peritoneal cavity $^{[41]}$. Hence, the omentum makes an ideal flap for draining stagnant lymphatic fluid. The large size of the omentum can be used to cover larger areas and can even be divided into two to treat bilateral lymphoedema ${ }^{[42]}$. Raising the omental flap via a laparotomy can result in abdominal wound infections, hernias, prolonged ileus and bowel obstruction ${ }^{[43]}$. Harvesting the omental flap by laparoscopy obviates the disadvantages that a laparotomy has by decreasing pain, discomfort, blood loss, wound infections, chest infections, prolonged ileus and bowel obstruction and deep vein thrombosis ${ }^{[4]}$. The major drawback of this flap is the need for laparoscopy and poor aesthesis associated with the bulk and skin grafting of the flap.

The jejunal mesenteric lymph node flap is also a very good option to avoid donor site lymphoedema following lymph node transfer. The mesentery in the jejunum is preferred to that in the ileum as it has more lymph nodes in the flap. The longest loop of the third part of the jejunum is identified and a flap based on the second, third or fourth mesenteric branch of the superior mesenteric artery is designed ${ }^{[45]}$. The proximal segment has significantly more lymph nodes than the other segments do, and the flap is raised preferably close to the root of the mesentery. To avoid a risk of internal hernia, only the anterior peritoneum containing the mesenteric lymph nodes and adjacent branches of the superior mesenteric vessels is raised, leaving behind the posterior peritoneum intact. Disadvantages of the flap include the risk of injury to the viscera, bowel adhesions, internal hernia and the need for bowel resection in case of bowel ischaemia.

The outcomes of VLNTs seem very promising according to various reports ${ }^{[45-47]}$. However, one needs to take steps to avoid complications. The paramount concern that one has while raising these flaps is not 
only to avoid damage to the vital structures, but also to avoid inducing lymphoedema in the donor limbs. Viitanen et al. ${ }^{[48]}$ demonstrated in their series that although none of their patients developed donor site lymphoedema, lymphoscintigraphy in over half the patients showed a slightly slower lymphatic flow in the donor limb compared to the non-operated limb. Donor site lymphoedema can be reduced by reverse lymphatic mapping ${ }^{[32]}$. The lymphatic drainage of the flap to be harvested and the potential donor site lymphoedema area are evaluated by two different methods. For example, before raising the DIEP flap containing the groin lymph nodes, ICG is injected intradermally in the lower abdomen and technetium injections in the first and second webspaces of the foot. A gamma probe is used to localise the lymph nodes draining the lower extremity which is avoided. Similarly, technetium can be injected into the hand and ICG injected into the back and lateral chest in cases of VNLT.

Postoperatively, the use of drains, antibiotics, anticoagulation and therapy is largely based on anecdotal experience and surgeon preference, as there is no uniform consensus on this subject, and a conclusive study can be difficult to devise considering the many variables and confounding issues involved. Compression over the flap is avoided for 2 to 3 weeks. Later, the patient is advised to wear compression garments throughout the day. Six months later, the patient can be reviewed, and if necessary, the excess fat in the limbs can be removed by liposuction.

\section{LIPOSUCTION}

In lymphoedema there is a physiological imbalance of blood flow and lymphatic drainage, resulting in the impaired clearance of lipids and the uptake by macrophages ${ }^{[49]}$. Besides, it is believed that chronic inflammation in lymphoedema results in adipose tissue hypertrophy ${ }^{[50]}$. Several studies have found an association with fat hypertrophy along with lymphatic stasis. The findings are indicated below:

1. In Crohn's disease, there was increased fat deposition in the areas of the bowel that were inflamed, suggesting that inflammation plays an important role in increasing fat ${ }^{[51]}$.

2. Analysis of the content of the aspirate from liposuction showed a high content of adipose tissue $(90 \%)^{[52]}$.

3. Dual energy X-ray absorptiometry in 18 women with post-mastectomy lymphoedema showed a significant increase in adipose tissue in the oedematous $\operatorname{limb}^{[53]}$.

4. Marked mononuclear cell response was found with adipogenesis in response to lymphatic fluid stasis ${ }^{[54]}$. 5. Lymphatic fluid stasis was shown to upregulate fat differentiation markers ${ }^{[55]}$.

The limbs affected with lymphoedema are swollen due to the accumulation of lymphatic fluid along with excess fat deposition. The accumulated lymphatic fluid can be decreased by conservative therapies such as complex decongestive therapy and controlled compression therapy along with microsurgical techniques such as LVA or VLNT. After lymphatic fluid has been decreased by the above procedures, the accumulated fat can be removed by liposuction. Liposuction is a method to remove fat and should be used only when the conservative or microsurgical techniques have been used before. The patient should be on compression garments. The absence of fluid collection is found when the limbs are not pitting even on applying pressure to them for a minute. Liposuction should only be done when there is no pitting and when the pitting cannot be reduced further even after regular physiotherapy ${ }^{[50]}$.

Under appropriate anaesthesia, power-assisted liposuction is used to reduce surgeon fatigue and also to facilitate liposuction especially in fibrous areas. Initially, the "dry technique" has been used ${ }^{[56]}$. To minimise blood loss, a combination of tourniquet and tumescence liposuction has been used ${ }^{[56]}$. A sterile tourniquet is tied proximally on the limb. Through appropriate 3-mm long incisions, liposuction cannulas 15 and $25 \mathrm{~cm}$ long and with diameters of 3 and $4 \mathrm{~mm}$ are used to aspirate the excess fat. A sterilised compressive dressing or a glove is worn, and the tourniquet is removed. The proximal part of the limb in which the tourniquet was removed is infiltrated with tumescence solution containing adrenaline and mild 
local anaesthetic, and liposuction is continued proximally. Finally, the proximal part of the arm is also compressed. The incisions are left open to drain. The hand is rested on a large pillow at the level of the heart. The next day, the dressings are replaced with compression garments.

After initial compression therapy, the garments need to be reassessed for loss of elasticity in the garment and for reduction in the size of the limb. This is very important especially in the first 3 months after surgery. The patient is assessed every 3 months for the first year to look out for change in the volume and also to inspect the condition of the compression garments. Maximum reduction of limb size is usually achieved in the upper limb at 3 months and in the lower limb at 6 months, but it may take longer. For best results, it is advised to wear the garment lifelong ${ }^{[57]}$. Hoffner et al ${ }^{[58]}$ have shown a mean 5 -year postoperative reduction of $117 \% \pm 26 \%$ in the limb with lymphoedema compared with the healthy arm.

\section{SURGICAL DEBULKING}

Surgical debulking of lymphoedema has been used for a long time. In spite of the popularity of surgical debulking decreasing due to the introduction of microsurgical techniques, surgical debulking remains the procedure of choice in carefully selected patients with Class III lymphoedema and skin changes, lymphoedema secondary to filariasis and in places where a microsurgical facility is unavailable.

In patients with filariasis, the adult worms of Wuchereria bancrofti, Brugia malayi and Brugia timori invade the lymphatic system and cause dilatation of the lymphatic channels, incompetence of the lymphatic valves and obliteration of the lymphatic channels ${ }^{[59]}$. This destruction of the lymphatic channels causes severe oedema and fibrosis in the limbs with resultant skin changes such as warty outgrowths, acanthosis and ulcers. When the limb and the toes become big, maintaining hygiene becomes difficult. This predisposes to many fungal infections in the interdigital spaces and fissures in the feet. These fungal infections act as entry points for many secondary bacterial infections, which produce acute dermatolymphangioadenitis ${ }^{[6]}$. Surveys estimate a frequency of 4.47 episodes of acute dermatolymphangioadenitis per year for bancroftian filariasis and 2.2 episodes for brugian filariasis ${ }^{[61]}$. Each episode of acute dermatolymphangioadenitis worsens the lymphoedema and produces more fibrosis, scarring and more swelling. Destruction of the lymphatic channels and severe fibrosis of the limbs precludes performing LVA and liposuction, respectively, in such patients. Furthermore, many patients with filarial lymphoedema and severe lymphoedema have many skin changes that are best treated by surgical debulking in the limbs. Microsurgical procedures such as LVA and VLNTs are generally beneficial in the early stages of the disease, when the lymphatics are relatively healthy and when the tissues are still soft and pliable. Chronic accumulation of lymphatic tissue in the subcutaneous tissues cause thickening of the skin, hypercellularity, progressive fibrosis, increased fat deposition and irreversible damage to the lymphatic vessels. For these patients with end-stage lymphatic disease, excisional surgical procedures remain the mainstay of patient management. Large folds of skin and subcutaneous tissue can be excised, which leads to improved outcomes. We will look at the different excisional techniques in detail.

\section{CHARLES PROCEDURE}

The Charles procedure is the prototype of all excisional procedures ${ }^{[62]}$. Although Sir Richard Henry Havelock Charles who described the Charles procedure, described the technique mainly for lymphoedema of the scrotum, the procedure bears his name for the excisional surgery for lymphoedema of the lower limbs as a result of series of questionable references ${ }^{[63]}$. The Charles procedure involves excision of the skin, subcutaneous tissue and deep fascia of the legs involved with lymphoedema and grafting the raw areas on the bare exposed muscle. This procedure is done in advanced lymphoedema with skin changes [Figure 2A-E]. After excision of the skin, due to the unavailability of subdermal lymphatic drainage, worse lymphoedema is expected distally in the foot. Severe secondary changes in the skin such as ulceration, 

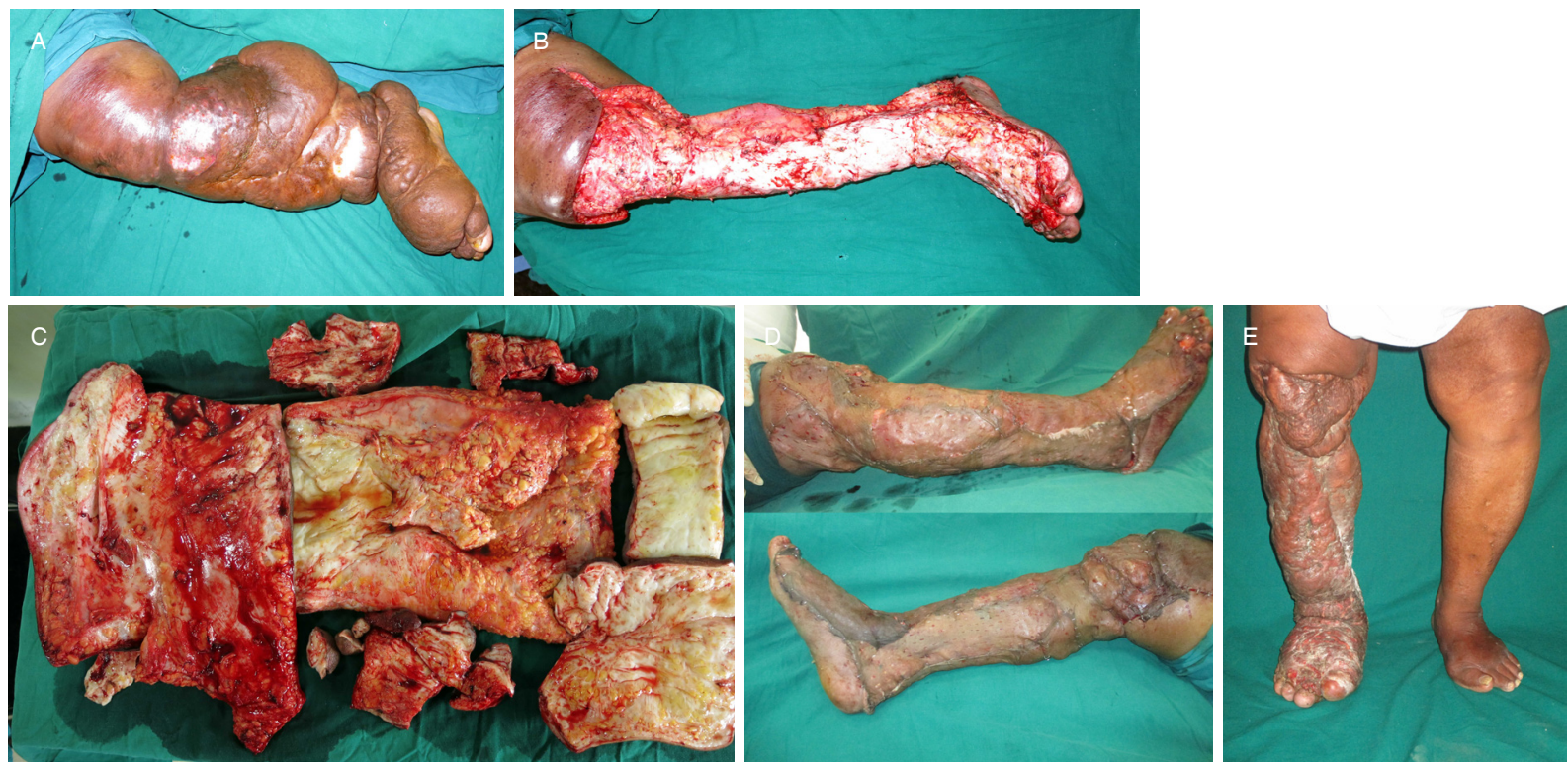

Figure 2. Charles procedure. A: pre-op photo of a patient with severe lymphoedema of the right leg; B: skin and subcutaneous tissue removed from the right leg; C: tissue removed; D: after skin grafting; E: long term result

papillomatosis, hyperkeratosis, weeping dermatitis and chronic cellulitis are commonly seen in the distal feet and toes ${ }^{[64]}$. Such skin changes in the toes can be very uncomfortable for the patient, and many patients may find it difficult to maintain personal hygiene. Karonidis et al. ${ }^{[65]}$ thought it advisable to preserve the toes if there was only swelling without previous cellulitis or verrucous hyperkeratosis and neither deformity nor osteomyelitis of the toes. Some surgeons have modified the original technique to preserve the deep fascia to improve lymphatic drainage of the leg. When this procedure is selected for the right patient, it results in considerable reduction in size, improvement in function and satisfactory results. However, due to the poor cosmesis, associated bottleneck deformity and distal lymphoedema, this procedure is not very commonly done. Van der Walt et al. ${ }^{[6]]}$ applied negative pressure wound therapy after excisional surgery to prepare the bed better for grafting. Negative wound pressure therapy is also commonly used after applying the grafts to keep the grafts in place and for better take. The Charles procedure can be associated with complications such as poor graft take, delayed healing, distal lymphoedema and recurrence of lymphoedema, especially at the foot, which may need resurfacing, regrafting and toe amputations. The patient needs to be taught good nail care and foot care, since infections in the nails can lead to repeated cellulitis and worsening lymphoedema.

\section{STAGED SUBCUTANEOUS EXCISION BENEATH SKIN FLAPS}

This surgery is performed in two stages both over the medial and laterals aspect of the limbs ${ }^{[67]}$. Usually the medial aspect of the limb is removed first as more tissue can be removed. An incision is made over the medial aspect of the limb. Flaps are raised on either side of the incision, and the excess tissue in the subcutaneous area is removed. The excess skin can be closed, trimmed or de-epithelialised. This is then repeated in the lateral aspect as well at least 3 months after the initial surgery.

\section{CHARLES PROCEDURE ALONG WITH VASCULARISED LYMPH NODE FLAP (CHEN-MODIFIED CHARLES PROCEDURE)}

To reduce the risks associated with lymphoedema, such as repeated cellulitis and recurrence and worsening of lymphoedema, VLNTs can be done along with the Charles procedure. While doing the Charles procedure, the superficial veins are to be kept intact so that they can be used as a recipient vein 
for the vascularised lymph node flaps. The vascularised lymph node flap is done distally in the limb and anastomosed to vessels around the ankle and the wrist. This helps to reduce the worsening distal oedema and recurrent cellulitis of the limbs ${ }^{[68]}$.

\section{RADICAL REDUCTION OF LYMPHOEDEMA WITH PRESERVATION OF PERFORATORS}

With knowledge of the anatomical position of the perforators of the limb and with various investigations such as handheld Doppler and CT scan, we are able to raise flaps with better vascularity. This helps us to excise tissue more confidently leaving behind good vascularised tissue. Accordingly, incisions in the limbs are made, and flaps based on the perforators are raised, while the excess tissue is radically removed. In the upper limb, the blood supply of the tissue left behind is based on the perforators from the brachial artery in the arm and from the radial, ulnar and posterior interosseous artery perforators. In the lower limb, the blood supply of the tissue left behind is mainly from the posterior tibial artery and peroneal artery. This technique is particularly useful for lower limb lymphoedema (52\% reduction) ${ }^{[69]}$ compared to upper limb lymphoedema $(15 \% \text { reduction })^{[70]}$.

\section{CONCLUSION}

The management of lymphoedema is an evolving science. Every patient with lymphoedema needs a detailed clinical evaluation, which may include investigations such as ICG lymphangiography to decide upon the best treatment. Understanding the benefits and limitations of various procedures will help us choose the optimal line of management for every patient. The results could be very gratifying both to the patient and the surgeon.

\section{DECLARATIONS}

\section{Authors' contributions}

Lead surgeon: Venkatramani $\mathrm{H}$

Preparation of manuscript and collection of data: Shanmugakrishnan RR

Assistant surgeon participated in most of the surgeries: Kumaran MS

Correction of manuscript and scientific advice: Sabapathy SR

\section{Availability of data and materials}

Not applicable.

\section{Financial support and sponsorship}

None.

\section{Conflicts of interest}

All authors declared that there are no conflicts of interest.

\section{Ethical approval and consent to participate}

Ethical approval obtained from the institutional review board (IRB). Written consent was obtained from all patients to take photographs and use their data for publication.

\section{Consent for publication}

The authors give consent for publication. Photographic consent was obtained for all patients.

\section{Copyright}

(c) The Author(s) 2020. 


\section{REFERENCES}

1. Silva AK, Chang DW. Vascularized lymph node transfer and lymphovenous bypass: novel treatment strategies for symptomatic lymphedema. J Surg Oncol 2016;113:932-9.

2. International Society of Lymphology. The diagnosis and treatment of peripheral lymphedema: 2013 Consensus Document of the International Society of Lymphology. Lymphology 2013;46:1-11

3. Tiwari A, Cheng KS, Button M, Myint F, Hamilton G. Differential diagnosis, investigation, and current treatment oflower limb lymphedema. Arch Surg 2003;138:152-61.

4. Lee BB. Contemporary issues in management of chronic lymphedema: personal reflection on an experiencewith 1065 patients. Lymphology 2005;38:28-31.

5. Shih YC, Xu Y, Cormier JN, Giordano S, Ridner SH, et al. Incidence, treatment costs, and complications of lymphedema afterbreast cancer among women of working age: a 2-year follow-up study. J Clin Oncol 2009;27:2007-14.

6. Koshima I, Inagawa K, Urushibara K, Moriguchi T. Supermicrosurgical lymphaticovenular anastomosis for the treatment of lymphedema in the upper extremities. J Reconstr Microsurg 2000;16:437-42.

7. Chang DW, Suami H, Skoracki R. A prospective analysis of 100 consecutive lymphovenous bypass cases for treatment of extremity lymphedema. Plast Reconstr Surg 2013;132:1305-14.

8. Suami H, Chang DW, Yamada K, Kimata Y. Use of indocyaninegreen fluorescent lymphography for evaluatingdynamiclymphatic status. Plast Reconstr Surg 2011;127:74-6e.

9. Yamamoto T, Kikuchi K, Yoshimatsu H, KoshimaI. Ladder-shaped lymphaticovenular anastomosis using multiple side-to-side lymphatic anastomoses for a leg lymphedema patient. Microsurgery 2014;34:404-8.

10. Koshima I, Narushima M, Mihara M, Yamamoto Y, Lida T. Treatments for leg lymphedema. J Jpn Soc Gynecol Oncol 2009;27:10-18.

11. Mazzei FG, Gentili F, Guerrini S, Cioffi Squitieri N. MR lymphangiography: a practical guide to perform it and a brief review of the literature from a technical point of view. Biomed Res Int 2017;2017:2598358.

12. Yamamoto T, Yoshimatsu H, Yamamoto N, Narushima M, Iida T, et al. Side-to-end lymphaticovenular anastomosis through temporary lymphatic expansion. PLoS One 2013;8:e59523.

13. Narushima M, Mihara M, Yamamoto Y, Iida T, Koshima I, et al. The intravascular stenting method for treatment of extremity lymphedema with multiconfiguration lymphaticovenous anastomoses. Plast Reconstr Surg 2010;125:935-43.

14. Yoshimatsu H, Yamamoto T, Narushima M, Iida T, Koshima I. The guide wire method: a new technique for easier side-to-end lymphaticovenular anastomosis. Ann Plast Surg 2014;73:231-3.

15. Yamamoto T, Chen WF, Yamamoto N, Yoshimatsu H, TashiroK, et al. Technical simplification of the supermicrosurgical side-to-end lymphaticovenular anastomosis using the parachute technique. Microsurgery 2015;35:129-34.

16. Mihara M, Hara H, Iida T, Todokoro T, Yamamoto T, et al. Antegrade and retrograde lymphatico-venous anastomosis for cancer-related lymphedema with lymphatic valve dysfuction and lymphatic varix. Microsurgery 2012;32:580-4.

17. Chen WF, Yamamoto T, Fisher M, Liao J, Carr J. The "octopus" lymphaticovenular anastomosis: evolving beyond the standard supermicrosurgical technique. J Reconstr Microsurg 2015;31:450-7.

18. Scaglioni MF, Fontein DBY, ArvanitakisM, Giovanoli P. Systematic review of lymphovenous anastomosis (LVA) for the treatment of lymphedema. Microsurgery 2017;37:947-53.

19. Chen WF, Zhao H, Yamamoto T, Hara H, Ding J. Indocyanine green lymphographic evidence of surgical efficacy following microsurgical and supermicrosurgical lymphedema reconstructions. J Reconstr Microsurg 2016;32:688-98.

20. Soran A, D’Angelo G, Begovic M, Ardic F, Harlak A, et al. Breast cancer-related lymphedema--what are the significant predictors and how they affect the severity of lymphedema? Breast J 2006;12:536-43.

21. Yamamoto T, Koshima I. Supermicrosurgical anastomosis ofsuperficial lymphatic vessel to deep lymphatic vessel for a patientwith cellulitis-induced chronic localized leg lymphedema. Microsurgery 2015;35:68-71.

22. Chang DW, Masia J, Garza R, Skoracki R, Neligan PC. Lymphedema: surgical and medical therapy. Plast Reconstr Surg 2016;138:209-18S.

23. Schaverian MV, Badash I, Patel KM, Selber JC. Vascularized lymph node transfer for lymphedema. Semin Plast Surg 2018;32:28-35.

24. Honkonen KM, Visuri MT, Tervala TV, Halonen PJ, Koivisto M, et al. Lymph node transfer and perinodal lymphatic growth factor treatment for lymphedema. Ann Surg 2013;257:961-7.

25. Saaristo AM, Niemi TS, Viitanen TP, Tervala TV, Hartiala P, et al. Microvascular breast reconstruction and lymph node transfer for postmastectomy lymphedema patients. Ann Surg 2012;255:468-73.

26. Lin $\mathrm{CH}$, Ali R, Chen SC, Wallace $\mathrm{C}$, Chang $\mathrm{YC}$, et al. Vascularized groin lymph node transfer using the wrist as a recipient site for management of postmastectomy upper extremity lymphedema. Plast Reconstr Surg 2009;123:1265-75.

27. Patel KM, Lin CY, Cheng MH. From theory to evidence:Long-term evaluation of the mechanism of action and flapintegration of distal vascularized lymph node transfers. J Reconstr Microsurg 2015;31:26-30.

28. Suami H, Chang DW. Overview of surgical treatments for breastcancer-related lymphedema. Plast Reconstr Surg 2010;126:1853-63.

29. Warren AG, Brorson H, Borud LJ, Slavin SA. Lymphedema: a comprehensive review. Ann Plast Surg 2007;59:464-72.

30. DiSipio T, Rye S, Newman B, Hayes S. Incidence of unilateral armlymphoedema after breast cancer: a systematic review and metaanalysis. Lancet Oncol 2013;14:500-15.

31. Van der Ploeg IM, Kroon BB, Valde's Olmos RA, NiewegOE. Evaluation of lymphatic drainage patterns to the groinand implications for the extent of groin dissectionin melanoma patients. Ann Surg Oncol 2009;16:2994-9.

32. Dayan JH, Dayan E, Smith ML. Reverse lymphatic mapping: a new technique for maximizing safety in vascularized lymph node transfer. Plast Reconstr Surg 2015;135:277-85. 
33. Mardonado AA, Chen R, Chang DW. The use of supraclavicular freeflap with vascularized lymph node transfer for treatment of lymphedema: a prospective study of 100 consecutive cases. J Surg Oncol 2017;115:68-71.

34. Sapountzis S, Singhal D, Rashid A, Ciudad P, Meo D, et al. Lymph node flap based on the right transverse cervical artery as a donor site for lymph node transfer. Ann Plast Surg 2014;73:398-401.

35. Ooi ASH, Chang DW. 5-step harvest of supraclavicular lymph nodes as vascularized free tissue transfer for treatment of lymphedema. J Surg Oncol 2017;115:63-7.

36. Bank J, Chang DW. Microsurgical procedures: vascularized lymph node transfer from the supraclavicular region. Principles and practice of lymphedema surgery. Philadelphia, PA: Elsevier; 2016. pp. 148-54.

37. Martin D, Pascal JF, Baudet J, Mondie JM, Farhat JB, et al. The submental island flap: anew donor site. Anatomy and clinical applications as a free orpedicled flap. Plast Reconstr Surg 1993;92:867-73.

38. Cheng MH, Patel KM. Micro surgical procedures: vascularized lymph node transfer from the submental region. Principles and practice oflymphedema surgery. Philadelphia, PA: Elsevier; 2016. pp. 138-47.

39. Cheng MH, Huang JJ, Nguyen DH, Saint-Cyr M, Zenn MR, et al. A novel approach to the treatment of lower extremity lymphedema by transferring a vascularized submental lymph node flap to the ankle. Gynecol Oncol 2012;126:93-8.

40. Barreiro GC, Baptista RR, Kasai KE, dos Anjos DM, Busnardo Fde F, et al. Lymph fasciocutaneous lateral thoracic artery flap: anatomical study and clinical use. J Reconstr Microsurg 2014;30:389-96.

41. Nguyen AT, Suami H. Laparoscopic free omental lymphatic flap for the treatment of lymphoedema. Plast Reconstr Surg 2015;136:114-8.

42. Nguyen AT, Suami H, Hanasono MM, Womack VA. Long term outcomes of the minimally invasive free vascularised omental lymphatic flap for the treatment of lymphedema. J Surg Oncol 2017;115:84-9.

43. Hultman CS, Carlson GW, Losken A, Jones G, Culbertson J, et al. Utility of the omentum in the reconstruction of complex extraperitoneal wounds and defects. Donor site complications in 135 patients from 1975 to 2000. Ann Surg 2002;235:782-95.

44. Lasso JM, Pinilla C, Castellano M. New refinements in greater omentum free flap transfer for severe secondary lymphedema surgical treatment. Plast Reconstr Surg Glob Open 2015;3:e387.

45. Coriddi M, Wee C, Meyerson J, Eiferman D, Skoracki R. Vascularized jejunal mesenteric lymph node transfer: a novel surgical treatment for extremity lymphedema. J Am Coll Surg 2017;225:650-7.

46. Becker C, Vasile JV, Levine JL, Batista BN, Studinger RM, et al. Microlymphatic surgery for the treatment of iatrogenic lymphedema. Clin Plast Surg 2012;39:385-98.

47. Basta MN, Gao LL, Wu LC. Operative treatment of peripheral lymphedema: a systematic meta-analysis of the efficacy and safety of lymphovenous microsurgery and tissue transplantation. Plast Reconstr Surg 2014;133:905-13.

48. Viitanen TP, Mäki MT, Seppänen MP, Suominen EA, Saaristo AM. Donor-site lymphatic function after microvascular lymph node transfer. Plast Reconstr Surg 2012;130:1246-53.

49. Brorson H. Liposuction in arm lymphedema treatment. Scand J Surg 2003;92:287-95.

50. Clayton DN, Clayton JN, Lindley TS, Clayton JL. Large volume lipoplasty. Clin Plast Sur 1989;16:305-12.

51. Borley NR, Mortensen NJ, Jewell DP, Warren BF. The relationship between inflammatory and serosal connective tissue changes in ileal Crohn's disease: evidence for a possible causative link. J Pathol 2000;190:196-202.

52. Brorson H, Aberg M, Svensson H. Chronic lymphedema and adipocyte proliferation: clinical therapeutic implications. Lymphology 2004;37:153-5.

53. Brorson H, Ohlin K, Olsson G, Karlsson MK. Breast cancer related chronic arm lymphedema is associated with excess adipose and muscle tissue. Lymphat Res Biol 2009;7:3-10.

54. Zampell JC, Aschen S, Weitman ES, Yan A, Elhadad S. et al. Plast Reconstr Surg 2012;129:825-34.

55. Aschen S, Zampell JC, Elhadad S, Weitman E, De Brot M, et al. Regulation of adipogenesis by lymphatic fluid stasis:part II. Expression of adipose differentiation genes. Plast Reconstr Surg 2012;129:838-47.

56. Wojnikow S, Malm J, Brorson H. Use of a tourniquet with andwithout adrenaline reduces blood loss during liposuction for lymphoedema of the arm. Scand J Plast Reconstr Surg Hand Surg 2007;41:243-9.

57. Schaverien MV, Munnoch DA, Brorson H. Liposuction treatment of lymphedema. Semin Plast Surg 2018;32:42-7.

58. Hoffner M, Ohlin K, Svensson B, Manjer J, Hansson E, et al. Liposuction gives complete reduction of arm lymphedema following breast cancer treatment-A 5-year prospective study in 105 patients without recurrence. Plast Reconstr Surg Glob Open 2018;6:e1912.

59. Shenoy RK. Clinical and pathological aspects of filarial lymphedema and its management. Korean J Parasitol 2008;46:119-25.

60. Shenoy RK, Suma TK, Rajan K, Kumaraswami V. Prevention of acute adenolymphangitis in brugian filariasis: comparison of the efficacy of ivermectin and diethylcarbamazine, each combined with local treatment of the affected limb. Ann Trop Med Parasitol 1998;92:587-94.

61. Panicker KN, Sebasan S. Socioeconomic perspectives. Miscellaneous publications of VCRC. 1990;16:42-7.

62. Charles RH. Elephantiasis scroti. In: Latham AC, English TC, editor. A system of treatment. London: Churchill; 1912.

63. Dumanian GA, Futrell JW. The Charles procedure: misquoted and misunderstood since 1950. Reconstr Surg 1996;98:1258-63.

64. Taylor GW. Surgical management of primary lymphoedema. Proc R Soc Med 1965;58:1024-6.

65. Karonidis A, Chen HC. Preservation of toes in advanced lymphedema: an important step in the control of infection. Ann Plast Surg 2010;64:446-50.

66. van der Walt JC, Perks TJ, Zeeman BJ, Bruce-Chwatt AJ, Graewe FR. Modified Charles procedure using negative pressure dressings for primary lymphedema: a functional assessment. Ann Plast Surg 2009;62:669-75.

67. Miller TA, Wyatt LE, Rudkin GH. Staged skin and subcutaneous excision for lymphedema: a favourable report of long-term results. Plast Reconstr Surg 1998;102:1486-98.

68. Sapountzis S, Ciudad P, Lim SY, Chilgar RM, Kiranantawat K, et al. Modified Charles procedure and lymph node flap transfer for advanced 
lower extremity lymphedema. Microsurgery 2014;34:439-47.

69. Salgado CJ, Mardini S, Spanio S, Tang WR, Sassu P, et al. Radical reduction of lymphedema with preservation of perforators. Ann Plast Surg 2007;59:173-9.

70. Salgado CJ, Sassu P, Gharb BB, Spanio di Spilimbergo S, Mardini S, et al. Radical reduction of upper extremity lymphedema with preservation of perforators. Ann Plast Surg 2009;63:302-6. 